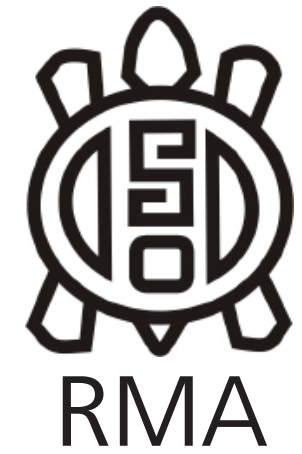

Antropología Social

\title{
El/la antropólogo/a en la aldea de la Mediación de conflictos. Un aporte para la problematización de las relaciones entre Estado, mediación de conflictos y Antropología
}

\author{
The anthropologist in the village of Conflict Mediation. A contribution \\ to the problematization of relations between State, conflict \\ mediation and Anthropology
}

Mariana Inés Godoy*, Juan Pablo Matta** y Kátia Sento Sé Mello***

${ }^{*}$ CONICET. Instituto de Investigaciones en Ciencias Sociales y Humanidades. CONICET- Universidad Nacional de Salta. E-mail: mariananes@gmail.com

***Grupo de Estudios Socioculturales del Conflicto. Facultad de Ciencias Sociales de Olavarría. Universidad Nacional del Centro de la Provincia de Buenos Aires. E-mail: jpmatta@soc.unicen.edu.ar

\begin{abstract}
**Programa de Pós Graduação em Serviço Social/UFRJ, Instituto Nacional de Estudos Comparados em Administração Institucional de Conflitos/Universidad Federal Fluminense y Núcleo de Estudos da Cidadania, Conflitos e Violência Urbana/Universidad Federal de Rio de Janeiro. E-mail: ksemello@gmail.com
\end{abstract}

\begin{abstract}
Resumen
En el presente artículo nos proponemos reflexionar sobre la mediación de conflictos en su vinculación compleja con la dimensión de lo estatal y específicamente con el campo estatal de la administración de conflictos. Para ello recuperaremos tres experiencias de investigación etnográfica desarrolladas durante los últimos años por los autores en distintos contextos (Rio de Janeiro en comparación con la ciudad de Buenos Aires, Salta y Olavarría). Lo que se denomina movimiento de mediación supone una articulación singular entre organismos judiciales, gubernamentales de distinta escala, el mundo de las ONGs, agencias internacionales, entre otros y una disputa permanente por la afirmación de este sistema se monta alrededor de darle mayor estatalidad al mismo. En segundo lugar, con el objetivo de dar cuenta de las características institucionales de este singular mundo repasaremos distintos aspectos vinculados a nuestros trabajos como antropólogos/as en dicho contextos. Proponemos que la experiencia etnográfica en dichos escenarios exige distintas disposiciones por parte del investigadorla que mediante un ejercicio reflexivo pueden tornarse potentes vías de acceso a los mundos que se analizan.
\end{abstract}

Palabras clave: Mediación de conflictos; Estado; Administración de conflictos; Etnografía; Reflexividad.

\begin{abstract}
In this article, we propose to reflect on conflict mediation in its complex relationship with the government dimension and specifically with the government field of conflict management. To do this, we will recover three ethnographic research experiences developed in recent years by the authors in different contexts (Rio de Janeiro, compared to the city of Buenos Aires, Salta and Olavarría). What is called the mediation movement implies a particular coordination between government agencies, courts of different scales, the world of NGOs, international organizations, among many others and a permanent dispute over the affirmation of this system is mounted around Give it more statehood. Secondly, with the objective of dealing with the institutional characteristics of this particular world, we will review different aspects related to our work as anthropologists in such contexts. We propose that the ethnographic experience in these scenarios requires different provisions on the part of the researcher that through a reflexive exercise can become powerful access roads to the worlds being analyzed.
\end{abstract}

Keywords: Conflict mediation; Government; Conflict management; Ethnography; Reflexivity.

La mediación de conflictos se enmarca en los denominados Métodos Alternativos de Resolución de Conflictos (ADR por su siglas en inglés), entre los que se encuentran la negociación, facilitación, conciliación y arbitraje, donde un tercero neutral - el/la mediador/a- utilizando el método autocompositivo y la comunicación no violenta (Rosemberg, 2006) colabora en la comunicación de las partes en conflicto en el horizonte de una solución 
que represente un tiempo menor que el que llevan los procesos judiciales adversariales tradicionales. El desarrollo de este dispositivo tanto en Brasil como en Argentina se enmarca en un esfuerzo a escala regional de desformalización de la justicia (Matta y Godoy, 2016) y de ampliación del acceso de la población al sistema judicial (Azevedo, 2000; Amorim, Kant De Lima y Burgos, 2003: 19-52). A la vez, esta modalidad trata principalmente con conflictos considerados "menores" (Cardoso de Oliveira, 2004; Simião, Duarte, Carvalho y Davis, 2010; Merry, 1995) por el sistema judicial, que involucran por lo general relaciones de proximidad (parentesco, vecindad, amistad), y sobre todo en los casos penales, situaciones de violencia interpersonal (Godoy, 2016). Se trata de una forma de administrar los conflictos que, según argumentan sus partidarios, propicia una dimensión pedagógica de la percepción del conflicto entendiendo a éste como una instancia propositiva y de aprendizaje en la que los conflictos perderían su connotación negativa. De esta forma, muchos investigadores han destacado los aspectos transformativos de la mediación (Bush y Folger, 1996) y el desafío que impone a las estructuras tradicionales, jerárquicas e inquisitoriales que organizan las instituciones estatales. (Sinhoretto, 2011; Mello y Lupetti Baptista, 2011, Mello 2016, Mello, Lupetti Baptista y Filpo 2018).

Entre mucho otros, un rasgo común de las tres investigaciones que se recuperan en el presente artículo es la presencia de lo estatal en cada uno de los ámbitos trabajados. Tanto en el caso de la mediación judicial de conflictos estudiada por Mello (Mello, 2011, Mello y Luppetti Baptista 2016, Mello et al, 2018) en Río de Janeiro y en la ciudad de Buenos Aires, la mediación penal en la provincia de Salta analizada por Godoy (2016 a y b) como la mediación comunitaria trabajada por Matta (Matta, 2016; 2020; Godoy y Matta, 2018; Matta y Godoy, 2016) en la ciudad bonaerense de Olavarría, la cuestión estatal aparece como un aspecto central en relación a las estrategias de afirmación, desarrollo y expansión seguidas por los partidarios de la mediación en cada contexto. Como resultado de esto, si bien lo que se ha denominado el movimiento de la mediación no supone en sí una estructura plenamente estatal, en todos los casos la posibilidad de hacerse con porciones de Estado (Fassin, 2003) y del maná estatal para desarrollar los dispositivos de mediación ha estructurado las dinámicas de cada una de esas experiencias.

En los tres casos los dispositivos que hemos trabajado se despliegan en y dependen de ámbitos estatales precisos (Ministerio Público, Poder Judicial, Municipalidades); reciben financiamiento también estatal y se inscriben en organigramas gubernamentales en el marco de los cuales se definen los alcances y límites de los mismos. De igual modo, la preocupación por el ordenamiento legal de estas prácticas es otra de sus características, lo que amplía aún más las presencias estatales (Manzano y Ramos 2015) de estas experiencias.
Existe sin embargo cierta discontinuidad entre el mundo de la mediación de conflictos y lo que podríamos delimitar como el campo estatal que invita a pensar estas relaciones en sus complejas articulaciones, disputas y dinámicas de diferenciación. Estas características moldean un tipo de estatalidad en el campo de la mediación sumamente dinámica que se parece más a un dialogo -o disputa- entre un movimiento (la mediación) y las diversas estatalidades en las cuales este se despliega, que a la identificación directa de uno con otro.

Por otra parte, el Estado aparece en la perspectiva de los actores de la mediación como una fuente no sólo de recursos materiales sino además fundamentalmente simbólicos, es decir, de reconocimiento oficial de su eficacia relativa en relación a otros dispositivos de la administración de conflictos ${ }^{1}$. La mediación se caracteriza en los contextos analizados por un esfuerzo de institucionalización y en ese proceso la estatalidad aparece como la fuente de legitimación principal ${ }^{2}$. En cualquier circunstancia, la lucha por la afirmación del campo de la mediación supone un ejercicio reflexivo por parte de los actores en relación al Estado y a las posibles relaciones entre éste y el propio movimiento de la mediación. Tal como señalan Manzano y Ramos (2015):

Para los mismos movimientos sociales, el entendimiento del Estado es un punto de partida a la hora de definir sus estrategias: como formas autónomas o de autodeterminación ante el Estado, como acciones del Estado contra el Estado, como participaciones dentro del Estado para transformar progresivamente el Estado, o como profundizaciones desafiantes de los mismos argumentos y fundamentos del Estado." (p. 9).

Creemos que este es un rasgo central en la medida que la mediación se configura como un ámbito en si mismo que logra inscribirse en esferas estatales sin perder completamente su autonomía como movimiento. Tal como trataremos de mostrar, los dispositivos y actores estatales de la mediación disponen de un espacio social relativamente autónomo ${ }^{3}$ en el cual, y desde el cual, se

\footnotetext{
${ }^{1}$ Bourdieu, en su texto más próximo a la lógica económica del Estado, describe a éste como un campo de poder dentro del cual se lucha por el poder sobre el Estado (Bourdieu, 1997: 100).

2 Por estatalidad entendemos la capacidad de las prácticas de gobierno (Foucault, 2006) de conducir conductas y establecer acuerdos sociales y morales (Bourdieu, 2014) sobre aquello que entra en la órbita del Estado, del interés público en oposición a lo que es privado. Estos acuerdos son también sobre las lógicas en que no sólo el conflicto es regulado y administrado sino sobre cómo éste puede expresarse. En palabras de Bourdieu: "para que sea posible el conflicto mismo sobre el mundo social, se necesita una especie de acuerdo sobre los territorios de desacuerdo y sobre los modos de expresión del desacuerdo" (pág. 12). Ese es uno de los fundamentos del Estado.

3 Bourdieu trabajó la idea de autonomía para el concepto de Estado refiriéndose al proceso histórico de parcial autonomía de la esfera política respecto de las relaciones de parentesco, las
} 
proyectan las relaciones con el primero al mismo tiempo que sirve de soporte para contener las inestabilidades que las experiencias estatales presentan para los mismos.

Tomando estas observaciones como punto de partida realizaremos primero una breve caracterización del mundo de la mediación en Argentina y Brasil focalizando en sus articulaciones y diferencias con lo que podríamos englobar como campo estatal y más particularmente con el campo estatal de la administración de conflictos. En segundo lugar, mediante un ejercicio reflexivo, se recuperarán una serie de instancias del trabajo de campo realizado por los autores en las cuales se expresan distintos modos en que la singularidad de la relación Estado-mediación moldeó la propia experiencia etnográfica. Finalmente, retomando los puntos anteriores, se ensayarán algunas consideraciones en torno al potencial que la reflexividad sobre este tipo de experiencias contienen para pensar lo estatal incluso cuando este no haya sido el objeto central de ninguna de las tres investigaciones.

\section{1- La constitución del campo de la Mediación de conflictos y la cuestión del Estado en Argentina y Brasil}

Lo que llamamos el campo de la mediación de conflictos ${ }^{4}$ desborda lo estatal pero a la vez está inserto y conectado con dicha arena. Al mismo tiempo excede lo judicial pero está profundamente conectado con este campo. Forma parte entonces de lo que Jacqueline Sinhoretto (2010) Ilamó "campo estatal de la administración de conflictos" aunque creemos necesario profundizar en esta caracterización. Esta socióloga y antropóloga brasilera, a partir de una combinación de tradiciones teóricas que abrevan en Pierre Bourdieu, Michel Foucault y aportes de antropólogos como Louis Dumont, y los brasileños Roberto DaMatta y Roberto Kant de Lima, Ilama "campo estatal de la administración de conflictos" a un espacio de concurrencias de corporaciones de sistemas de justicia, formas de saber y hacer y rituales de administración de conflictos que tienen a la vez una relación de disputa y jerarquía entre sí y producen efectos diversos.

En este campo, a diferencia del campo jurídico en un sentido mas clásico, aparecen posiciones institucionales novedosas y relativamente subalternas tales como la de los mediadores o la de los legos del derecho a medida que se universalizan reformas judiciales que proponen ritos informales, orales y participativos. Siguiendo una perspectiva foucaultiana, esta noción permite además pensar lógicas de funcionamiento y formas de circulación

relaciones económicas o religiosas. Nosotros más bien nos referimos a la autonomía de diferentes burocracias al interior de la misma organización estatal.

${ }^{4}$ Seguimos de manera general la noción bourdiana de campo, que nos ayuda a pensar los esfuerzos de la práctica por delimitar lo específico de un espacio social y a la vez de situar esa especificidad a partir de luchas de afirmación, tensiones y relaciones de fuerza. Ver Bourdieu (Cfr. Bourdieu, 1990 y 1995) del poder en los sistemas jurídicos que están al mismo tiempo, dentro y fuera del Estado. Por otro lado, se acepta desde este enfoque que cualquier técnica de administración es politizada, reconociendo la politización del campo jurídico estatal (Sinhoretto, 2010: 110). La autora entiende además que en las últimas décadas es un campo que se ha vuelto más politizado, pues ha sido tensionado tanto por movimientos de reformas en la justicia y de la policía, como por activismos por derechos colectivos $^{5}$.

La noción de movimiento con la que la mediación se presenta a sí misma nos llama la atención también sobre la relación ambivalente entre lo que está a la vez dentro y fuera del Estado. En la literatura académica del propio campo, proveniente especialmente del mundo anglosajón, se usa recurrentemente este concepto aunque no se lo define con demasiada precisión. Éste parece aludir por un lado al movimiento desde la justicia tradicional, los tribunales, el litigio o la adjudicación hacia formas "alternativas" de tratamiento de las disputas incluyendo dispositivos como la mediación, el arbitraje, la negociación e incluso el juicio por jurados, entre otros (Fleming, 1985). Y por el otro hace alusión a una significativa acumulación de actores heterogéneos en la conformación de ese movimiento, tales como programas públicos y privados, socios de ADR (Alternative Dispute Resolution) en las principales firmas de abogados, organizaciones privadas dedicadas a la promoción del sistema, servicio de mediación de las Cortes, escuelas de formación profesional, incremento de la asistencia a capacitaciones y la proliferación de revistas, boletines, libros y artículos sobre negociación y mediación. (McEwen, 1988; Thomas, 2016).

Para el caso argentino hemos descripto el proceso de conformación del campo de la mediación en tanto movimiento (Matta y Godoy, 2016), mostrando que desde sus orígenes hacia la década del ' 80 y ' $90^{6}$, se produce una relación de imbricación entre actores heterogéneos

\footnotetext{
${ }^{5}$ Dos características adicionales de ese campo, remontándose a los aportes de Louis Dumont, Roberto DaMatta y contemporáneamente Roberto Kant de Lima son, según propone la autora: 1) la coexistencia de una lógica jurídica jerárquica y una lógica igualitaria; 2) el hecho de que la informalidad e incluso la ilegalidad son parte estructurante de este campo

${ }^{6}$ El 4 de octubre de 1995 el Parlamento argentino sancionó la Ley 24.573 "Mediación y Conciliación" poniendo en vigencia en el ámbito de la justicia nacional el denominado sistema de mediación previa obligatoria. En 1992 se había declarado por un decreto presidencial de interés nacional la institucionalización y el desarrollo de la mediación como método no adversarial de resolución de conflictos, encomendándose al Ministerio de Justicia de la Nación la formulación de proyectos legislativos y el dictado de normas de nivel reglamentario para la puesta en marcha de dicha institución. También, mediante ese decreto se creó el Cuerpo de Mediadores del Ministerio de Justicia. Anteriormente, según se pudo registrar, hacia 1989, un grupo de jueces argentinos había participado, junto a 120 magistrados de toda América, en un curso de la Escuela Judicial de Reno, Nevada, Estados Unidos donde tomaron contacto por primera vez con los sistemas de resolución alternativa de disputas.
} 
que provenían del campo judicial, el activismo de ONGs ligados a lo judicial, actores de promoción del crédito y gobierno internacional (Banco Mundial, Banco Interamericano de Desarrollo, FMI, Organización de Estados Americanos) y la iniciativa pública a través de la proliferación de normativas nacionales y provinciales.

Ese proceso inicial formaba parte de un paquete de reformas neoliberales del Estado donde resuenan conceptos como crisis y emergencia de los sistemas judiciales al igual que el proyecto ideológico para América Latina de buen gobierno o good governance. La apropiación y gestación local de esa experiencia fue diversa, al igual que desigual su desarrollo, según pudimos observar comparativamente para el caso de Salta y de Olavarría en el contexto de la provincia de Buenos Aires. Si bien no podremos desarrollar en el presente artículo en profundidad esas especificidades, cabe apuntar que por ejemplo la provincia de Salta suele presentarse y ser reconocida como "pionera" y "referente" a nivel nacional e internacional en materia de implementación de sistemas de mediación, logrando en pocos años un despliegue territorial amplio de este dispositivo bajo órganos como el Ministerio Público, el Poder Judicial, el Ministerio de Justicia del Ejecutivo provincial en relación con gobiernos municipales, y abarcando un conjunto diverso de conflictividades tanto de origen civil como penal. Este proceso, que además genera incesantemente nuevas iniciativas parece superar a veces las iniciativas de orden nacional, mostrando la importancia de la dimensión local para el desarrollo de ciertas políticas públicas. En Olavarría, una ciudad de escala mucho más pequeña (con 140000 mil habitantes) la mediación ha encontrado cobijo institucional en el estado municipal y puesto su rúbrica singular en conflictos de orden vecinal, que en otros distritos aparecen mucho menos delimitados y más mixturados con el tratamiento de disputas de origen familiar.

De este modo identificamos la existencia de una multiestatalidad o multiplicidad de niveles estatales e institucionales comprendidos en su emergencia y desarrollo. A la vez estos niveles se articulan y oponen con formas de mediación por fuera del estado, organizadas en estudios de mediación privados o alrededor de instituciones como los colegios profesionales. También la formación y actualización de la formación de mediadores, que es una exigencia permanente en el campo, se liga a fundaciones y ONG's de origen local o nacional como la misma Fundación Libra, Intermed o en su momento Mediando. En ocasiones los mediadores se pueden mover de un lugar a otro ante cambios de coyunturas de orden político o pertenecer al mismo tiempo al espacio público y privado. También hay que hacer notar que la noción de conflicto en oposición a nociones como litigio o delito, se transforma en una categoría amplia que va a permitir una intervención legítima cada vez mayor sobre una diversidad de disputas sociales ${ }^{7}$.

\footnotetext{
${ }^{7}$ Siguiendo a Bourdieu (2014) la capacidad de producir categorías y
}

En Brasil, la mediación de conflictos se establece tardíamente en comparación con Argentina. En el caso de la mediación judicial, hay un discurso que refuerza una especie de mito fundador en el ámbito del Tribunal de de Justicia de Rio de Janeiro, teniendo como marco el año 2009, cuando se realizó el primer curso formal de mediación judicial de conflictos. Este curso fue destinado a operadores del Tribunal de Justicia -magistrados y técnicos como asistentes sociales y psicólogos- así como invitados externos al Tribunal. Como ya fue demostrado por Mello y Lupetti Baptista (2011), en Brasil la implantación de la mediación y de la conciliación en los Tribunales Estatales de Justicia se extiende más allá de los mismos. Diversas organizaciones gubernamentales y no gubernamentales han tenido iniciativas y prácticas en el mismo sentido del caso de las Escuelas del Perdón y Reconciliación (ESPERE) de la Pontificia Universidad Católica de Rio de Janeiro; del Instituto Mediare: Diálogos y Procesos Decisórios, especializado en la "prevención, evaluación, administración y resolución de conflictos" con el objetivo de facilitar el diálogo y construir consensos en procesos decisorios. Otros institutos se crean y desarrollan en el nivel comunitario, aunque siguen teniendo referencia en los tribunales. Entre estos encontramos organizaciones no gubernamentales como el Balcão de Direitos, de Viva Rio; el Núcleo de Mediación de Carmo, en Olinda, en el nordeste do Brasil. Las autoras mencionadas consideran que esta diseminación de la mediación de conflictos parece haber constituido en Brasil un "campo de fuerzas" (Bourdieu, 1990), en el que diferentes percepciones, representaciones, propuestas y prácticas se tornan objeto de disputa delineando fronteras, tensiones y alianzas institucionales.

Otro aspecto destacado por Mello y Lupetti Baptista (2011) y Mello (2020) es aquello que la experiencia de implantación de la mediación judicial de conflictos en Brasil dice respecto a la forma en que fueron planteadas las propuestas institucionalizadas de implementación de centros de mediación de conflictos. Se habla así de "formas alternativas" de administración de conflictos. Sin embargo, el adjetivo "alternativo" siempre llamó la atención puesto que por ejemplo en Timor Oriental los procedimientos y técnicas inscriptos en la denominación mediación de conflictos son practicados por los ancianos de las aldeas, es decir, fuera de los tribunales (Simião, 2015). Se trata de la mediación tradicional de conflictos, donde la oralidad recibe especial atención. Las autoras indagan, por lo tanto, sobre cuál es el significado de la palabra "alternativo". ¿La mediación sería una alternativa al proceso judicial en si o su forma tradicional de administrar conflictos, basada en el privilegio del orden

clasificaciones sociales es uno de los actos y efectos más notorios del Estado, produciendo asimismo identidades sociales. Con la noción de "conflicto", el campo de la mediación está también pensando en un sujeto que no es ya el criminal o delincuente ni el transgresor de la norma sino una persona moral envuelta en disputas de intereses y de reconocimiento (Cardoso de Oliveira, 2004) en sus relaciones sociales más próximas. 
jurídico sobre el social? "Alternativo" parecía también, remitir a la existencia de varias opciones, siendo la mediación de conflicto una entre otras. Cuando se escribió esta reflexión en 2011, parecía que tal adjetivo tenía por referencia el propio proceso judicial y no una forma diferenciada de administrar conflictos. Asimismo tenía como punto central al propio juez, quien remitía a las personas al Centro de Mediación (Mello e Lupetti Baptista, 2011).

Como adelantamos, en Argentina la mediación de conflictos fue implantada en 1996, mientras que en Brasil recién en 2015 se aprobó la Ley 13.140, que dispone "la mediación entre particulares como medio de solución de controversias y sobre autocomposición de conflictos en el ámbito de la administración pública". Desde entonces, los tribunales de justicia adoptaron la denominación Método Consensual/adecuado de Solución de Conflictos, constituyendo los Núcleos Permanentes de Métodos Consensuales de Solución de Conflictos (NUPEMEC).

En la ciudad y provincia de Buenos Aires, la mediación es una práctica exclusiva de los abogados. En Salta, salvo en el ámbito del Poder Judicial, la ley provincial optó por la interdisciplinariedad, siendo para sus actores un logro y un valor. En Brasil la mediación judicial puede ser ejercida por personas que no fueron socializadas en la enseñanza jurídica. La exigencia para el desarrollo de la práctica de mediación es que los medidores hayan realizado estudios superiores completos y que cumplimenten algún curso de capacitación en mediación que sea reconocido por el Ministerio de Justicia a través del Consejo Nacional de Justicia, el cual dispone del reconocimiento de los cursos ofrecidos, ya sea por los tribunales de justicia o por instituciones particulares. En el ámbito de la denominada "mediación comunitaria" tanto en Rio de Janeiro como en Salta los mediadores no precisan contar con título universitario, no obstante, igual que los mediadores judiciales, les es exigido capacitarse en alguna formación homologada por el Ministerio da Justicia. En Rio de Janeiro, comparativamente con Buenos Aires, aunque los mediadores no sean actores del campo jurídico, (Mello, Lupetti Baptista, Filpo, 2018) cuestionan cual es la frontera entre lo judicial y lo extrajudicial y lo que pode ser considerado formal o informal en el contexto de la medición de conflictos.

A pesar de la gran producción académica sobre el tema en Brasil, la mediación de conflictos ocupa un lugar de "baja intensidad" en el campo estatal da gestión de conflictos (Sinhoretto, 2011) así como parece también ocupar un lugar de "baja intensidad" reflexiva en las Ciencias Humanas stricto sensu, mientras adquiere preeminencia en los estudios sociológicos del Derecho (Mello, 2020). En contraste, en Argentina los estudios sobre mediación en el campo de las Ciencias Sociales son todavía incipientes, a diferencia del desarrollo institucional mucho más denso.
La investigación sobre la mediación de conflictos en Brasil y Argentina indica que existe un discurso normativo y práctico entre los mediadores de conflictos en Brasil y una especie de creencia en la efectividad de la mediación (Matta y Godoy, 2018) en el caso de Argentina. Sin embargo, en Brasil, la investigación empírica ha demostrado que es un espacio de tensión y conflicto. Como se analizó en Mello y Lupetti Baptista, (2011) existen obstáculos para la implementación de la mediación de conflictos dentro del Sistema de Justicia Penal. Esto se vincula con el sistema procesal brasileño que concierne al contradictorio como principio y como lógica de estructuración del proceso judicial y se extiende a la concentración en la figura del juez para la conducción del proceso; la representación de que la mediación asociada a la idea de cultura de la paz puede minimizar la "cultura del litigio" en Brasil y a la idea de celeridad como objetivo del poder judicial (Mello e Lupetti Baptista, 2011).

Sinhoretto (2011) argumenta que, en Brasil, existe un consenso que se extendió hasta la década de 1990 en relación a una expansión de la Ley y el Poder Judicial en la sociedad brasileña y asimismo que los Juzgados de Pequeñas Causas serían una de las experiencias de afirmación de la ciudadanía y una demostración de su potencial transformador. Destacando el trabajo de Vianna et al (1999 citado por Sinhoretto, 2011: 135), Sinhoretto agrega que tal experiencia plantea la hipótesis del potencial ético-pedagógico de una cultura cívica de autocomposición promovida por los Tribunales Penales Especiales, aproximándose al modelo constitutivo de la tradición del Common Law.

\section{2- El antropólogo en la aldea de la Mediación}

En esta segunda parte procuraremos una descripción comparativa de nuestra peculiar relación como antropólogos/as en/con este campo de la Mediación tanto en la producción académica como bajo modalidades de transferencia-. Consideramos que esto puede indicar algo sobre las características de este universo estatal de la administración de conflictos relativamente novedoso y que tiene para si unas exigencias muy particulares de posicionamiento institucional. El carácter dinámico del Estado y las disputas de identidad de sus agentes van moldeando nuestra experiencia de trabajo antropológico, esto es, nuestras posibilidades de alianzas, compromisos y lealtades con ese campo y de delinear objetivos de conocimiento. Estas cuestiones así planteadas pueden hacer un aporte general para pensar las intrincadas relaciones entre antropólogx/antropología y Estado.

\subsection{Modos de acceso y permanencia en el campo}

Queremos aquí plantear en primer término cuál fue nuestra modalidad de acceso y permanencia en nuestros respectivos espacios empíricos de observación y posterior 
análisis. Esta cuestión no es menor. Muzzoppapa y Villalta (2011) en un texto que ya es una referencia para pensar el acceso a las burocracias ligadas a la administración de conflictos plantean la importancia estratégica de la intervención de relaciones personales intra o extrainstitucionales e incluso la inserción laboral para el acceso a estos universos más o menos herméticos. En segundo lugar, las autoras nos indican que los obstáculos para el acceso, en el caso que analizan, a ciertos documentos estatales, deben convertirse en "dato" antropológico considerando el contexto de relaciones en el que estos productos estatales se generan y circulan. Estos aspectos guardan coherencia con un modo de comprensión del Estado, al que adhieren las autoras. Es cierto, se trata de una ficción que guarda su eficacia en la imagen de un ente homogéneo y espectrante que se cierne desde fuera sobre las personas. Pero que también debe entenderse como una organización de seres humanos individuales conectados por un complejo sistema de relaciones y por la orientación de actores que cotidianamente hacen al Estado (Muzzoppapa y Villalta, 2011: 17).

En el caso de Matta, su trabajo en el terreno de la mediación comunitaria orientada a la gestión de conflictos vecinales, surgió en el año 2013 a partir de la demanda de la entonces directora del Centro Municipal de Relaciones Vecinales y Defensa del Consumidor de la ciudad de Olavarría, provincia de Buenos Aires. Después de su experiencia como tesista doctoral estudiando la conflictividad social derivada de intercambios mercantiles, es decir, en el área de consumo, esta directora solicitó que su participación se extendiera y centrara su atención en la más reciente innovación política de aquella oficina que era en cambio el área vecinal. Esta relación se formalizó bajo un acuerdo de colaboración entre la Facultad de Ciencias Sociales de la UNICEN y la Municipalidad de Olavarría en el cual se fijaban distintos objetivos orientados al trabajo conjunto en el área municipal de mediación comunitaria.

En el caso de Godoy, su acceso al entonces principal centro de Mediación penal en el área del Ministerio Público de Salta se logró a partir del contacto personal con un mediador de ese organismo y la habilitación de parte de su directora a una aproximación bastante abierta y flexible a un conjunto significativo de instancias de trabajo e interacción de los mediadores; esta relación de campo no se formalizó en estricto sentido, pero llegó a ser de un intercambio asiduo y a tener una duración de seis años hasta la actualidad.

Tanto en el caso de Matta como de Godoy, las personalidades de las directoras -mujeres- de estos centros jugaron un rol importante en esta forma de acceso y la calidad de los lazos establecidos con nuestros interlocutores y con la institución a lo largo del proceso de investigación.

La investigación en Rio de Janeiro se dio a partir de la inserción de Mello en el Tribunal de Justicia de Estado (TJ-RJ). Esta investigadora recibió una invitación para participar del curso de mediación judicial de conflictos que se desarrolló en enero de 2009 de parte de uno de los jueces responsables del tribunal de familia y de un psicólogo que ya actuaba como mediador en el mencionado tribunal. Ella fue presentada a éstos por un amigo común con quien entró en contacto a raíz de un conflicto experimentado por una pareja de amigos que se encontraba en proceso de divorcio y demandaba la intervención de una tercera persona que facilitara su comunicación. En la búsqueda de alguna alternativa que cubriera las expectativas de esta pareja, Mello llegó al mencionado juez y al mediador. Luego de participar de un módulo de 40 horas del curso de mediación judicial, la investigadora cursó el módulo de 24 horas de mediación familiar. A partir de entonces, se tornó mediadora voluntaria del TJ-RJ, lo que le permitió el acceso no solamente a ciertos documentos escritos y a los responsables de la implantación de este instituto en Brasil, sino también a otros mediadores y a las audiencias de mediación. En Buenos Aires su inserción en los ambientes de mediación de conflictos también fue fruto de una red de amigos en Brasil y en Argentina, algunos de los cuales actuaban como mediadores en la ciudad porteña.

En Rio de Janeiro Mello pudo actuar como mediadora voluntaria entre el segundo semestre de 2009 y noviembre de 2011. En esa ocasión, los requisitos exigidos para tal actuación eran: tener nivel universitario en cualquier área de conocimiento, especialmente en el de Ciencias Humanas; haber realizado los cursos ofrecidos en el Tribunal de Justicia y haber participado como observadora de audiencias de mediación antes de actuar como mediadora. En Buenos Aires, por no ser abogada ni estar registrada en la Dirección Nacional de Mediación y Métodos Participativos de Resolución de Conflictos del Ministerio de Justicia, no tuvo la habilitación para actuar como mediadora. Sin embargo, tuvo acceso a diversas sesiones de mediación, fundamentalmente en materia familiar, por conocer a la entonces directora de la Dirección Nacional de Mediación de Conflictos y por haber obtenido autorización de las partes. A pesar de haber podido observar sesiones de mediación penal en dos centros del Ministerio Público de la ciudad, la opción de concentrarse en las sesiones de mediación familiar se debió al hecho de constituir un campo equivalente al del trabajo de campo que ya venía desarrollando en Rio de Janeiro hacía casi tres años y, por eso, pasible de comparación.

Esta experiencia en ciertos casos, de un relativo fácil acceso y tejido de colaboraciones más o menos prolongadas en el tiempo sobre la que volveremos luego, también habla de las características de la cultura institucional que comprende a la mediación, donde encontramos presente el valor de la interdisciplinariedad y de la formación permanente de recursos humanos en 
el área. Donde la competencia entre distintos organismos institucionales que se orientan a similares cuestiones también anima a sus actores a receptar positivamente una propuesta de investigación social en ese campo. A la vez la relativa autonomía de sus unidades de funcionamiento, característica común a varias burocracias judiciales en Argentina (Godoy, 2016a), facilita un acercamiento sin grandes obstáculos administrativos o barreras de mando en el tejido burocrático.

En Brasil, a su vez, la experiencia de Mello encontró algunos obstáculos para ampliar su participación, especialmente en ocasión de un proyecto solicitado por el propio Ministerio de Justicia de observación de prácticas de mediación en Brasil, cuando otros investigadores de diferentes Estados del país también realizarían pesquisas de campo. En nombre de una justificación jurídica -el secreto de justicia- los jueces responsables de los Centros de Mediación estaduales se opusieron a la inclusión de observadores auxiliares de investigación que, no siendo mediadores, no fueran autorizados a desenvolver las investigaciones en las sesiones de mediación. En cuanto a la presencia de observadores en las audiencias en Buenos Aires, los mismos debían ser autorizados por las partes en conflicto, en el caso de Brasil, dependía de la autorización formal del magistrado responsable ${ }^{8}$.

La figura del antropólogo en ese universo institucional parecía sin duda atractiva. En ocasiones también la titulación de "doctor" o "licenciado" otorgaba una legitimidad adicional, coherente con un ámbito donde las formalidades y el status profesional suelen ser apreciadas. La imagen del antropólogo se asociaba de manera inicial al interés por captar cierta dimensión cultural -sin que esta noción estuviera del todo definida- de los conflictos, y por ejemplo, en el caso de Salta se apuntaba especialmente a los conflictos en el ámbito barrial: -este conflicto te va a interesar- solían esgrimir los mediadores.

¿Qué roles adoptamos en el campo? En el caso de Godoy los primeros años se trató de un rol de observadora de audiencias de mediación y en ocasiones de acompañamiento, también mediante observación, de las tareas de la mesa de entradas. Ese rol de algún modo está asimilado sin demasiados inconvenientes en este espacio institucional, pues los mediadores acostumbran a observar audiencias -sin intervenir- para su formación inicial. Matta en cambio desde el inicio participó de manera directa en las intervenciones en conflictos vecinales superponiéndose su rol como investigador antropólogo con la de los propios mediadores. Así por ejemplo participó de las visitas a las casas donde vivían las personas que habían hecho un requerimiento en la oficina o bien que habían sido indicados por otro vecino como parte de una situación que requería algún tipo de tratamiento. Mello, con su diploma de Mediadora en la ciudad de Rio de Janeiro también pudo observar e

\footnotetext{
${ }^{8}$ Sobre esto, ver Mello, 2016.
}

intervenir en las audiencias. De hecho como explicamos, esta fue la manera de participar en estas instancias en calidad de antropóloga.

La relativa duración de los vínculos con estas instituciones se alimentó también de relaciones de afecto y amistad genuina entre nosotrxs y varixs de Ixs mediadores, compartiendo no sólo instancias de trabajo, o espacios en la universidad, sino cumpleaños o celebraciones de fin de año. En contraste, en situaciones de trabajo cotidiano podíamos también ser tratados con la más cruel indiferencia de las burocracias.

\subsection{De intervenciones, docencia y colaboraciones "técnicas"}

Como adelantamos, la colaboración en procesos de mediación concretos fue un hecho real y más o menos constante en nuestras experiencias de investigación en este campo institucional. A esto se suman otras formas de articulación entre investigación antropológica y mediación que es útil desarrollar para luego reflexionar sobre sus alcances.

Matta empezó a ser consultado por medios de comunicación, por ONG's y actores políticos de Olavarría ante distintas situaciones de conflictividad social, siendo en particular requerido por el Ministerio de Defensa para colaborar en la resolución de un conflicto complejo en torno a la explotación minera de un predio que esta repartición estatal tenía en los alrededores de esa ciudad y que había llevado a un pequeño poblado a vivir una situación muy difícil. En el caso de Godoy, en los años de trabajo en ese campo existieron pequeñas formas de asistencia "técnica" en torno a iniciativas de la mediación penal, transferencias o diálogos colaborativos de naturaleza reticular, aunque no necesariamente bajo convenios formalizados. Lo que es bastante común en el trabajo etnográfico, no poder diferenciar del todo las situaciones propias del trabajo de campo de aquellas colaboraciones efectivas, demandadas por el propio proceso de interacción ${ }^{9}$, y en algunos casos, como el nuestro, formando parte de los objetivos específicos de la propuesta original de investigación ${ }^{10}$.

\footnotetext{
${ }^{9}$ Ver por ejemplo Fasano (2014). Su investigación sobre el chisme se prolongó a tareas de comunicación institucional y comunitaria en la organización barrial con la que entabló su vínculo de campo y a la elaboración de proyectos de extensión universitaria. Allí explica que: "no ignoro que la investigación constituye de por sí un modo de intervención en la vida cotidiana de la gente, si bien considero que esa dimensión de la investigación antropológica es poco problematizada en nuestro ámbito académico" (pp. 6).

10 Por ejemplo mientras hacía trabajo de campo esta antropóloga procuró emitir opinión, en el momento de su elaboración, sobre las categorías de clasificación de los conflictos para el registro informático; mantuvo un par de reuniones con la directora de Mediación mientras elaboraba un protocolo de actuación en mediación en casos de violencia de género; colaboró en la elaboración de una estructura de "sistematización" de los casos de Conflictos Públicos Complejos tomando como base una propuesta de una formadora que los mediadores estimaban. Hubo varios intentos de colaboraciones
} 
Fleicher propuso en este sentido la categoría de antropólogos anfibios, es decir, aquellas formas concomitantes de trabajo en la universidad y en la vertiente de intervención como ONGs, órganos de gobierno, asociaciones comunitarias, en proyectos de agencias internacionales, en escuelas indígenas, consejos de salud, etc. Indica que la antropología brasilera siempre tuvo esta característica aunque esta dimensión quedó casi siempre como una actividad implícita incluso para representación de los antropólogos y que requiere entonces mayor reflexión. Al menos en el campo de la antropología jurídica o del análisis de las formas institucionales de administración de conflictos al que estas tres experiencias que relatamos se adscriben, la intervención definida a veces como "extensión", otras como "transferencia" o "vinculación tecnológica" ha sido una constante formando parte en ocasiones de los objetivos de investigación declarados en los planes iniciales. ${ }^{11}$

Uno de los modos principales que encontramos de hacer devoluciones y alimentar diálogos entre nuestra producción académica y el campo del ejercicio de la mediación fue la oferta de cursos, capacitaciones y formación en general, activando las disposiciones existentes en este campo de producción de alianzas entre instituciones estatales, universidades y organizaciones de la sociedad civil. Y también encontrando un resquicio allí donde para la mediación la formación permanente es una exigencia y un valor profesional incluso inscripto en los reglamentos.

Hacia el año 2013 Matta fue parte, junto a dos mediadoras de la ciudad de Olavarría, de la coordinación y docencia en un Programa municipal de formación de mediadores comunitario $^{12}$ orientado a actores vecinales. Ese mismo año dirigió un proyecto de extensión universitaria en el ámbito de la UNICEN denominado Desarmando Conflictos orientado a la familiarización con los modos alternativos de resolución de disputas en contextos en donde existían antecedentes de uso de armas de fuego como modalidad de confrontación. Luego, en el año 2014 se inauguró la Diplomatura Superior en Educación para la Paz y el Abordaje de la Conflictividad Social en la

malogradas que no terminaron en productos ciertos, pero que sin embargo alimentaban una constante relación de intercambio y reconocimiento de un saber mutuo.

11 De hecho en Brasil el InEAC al que pertenece Mello, tiene una tradición ya consolidada de articulación entre academia y organismos públicos y privados en el campo de las políticas públicas de administración de conflictos. Tal vez en Argentina esta experiencia es aún más incipiente y de hecho organismos de financiamiento de investigación como CONICET están exigiendo cada vez más mostrar la capacidad de transferencia tecnológica y estratégica de los conocimientos producidos por las Ciencias Sociales, generando todo un debate al interior del propio campo científico alrededor de la "utilidad" y validez de la ciencia "básica" y "aplicada".

12 "Programa Comunitario Municipal Abriendo Ventanas" orientado a la formación de mediadores comunitarios en ocho Servicios Territoriales en el distrito de Olavarría. Decreto Municipal $N^{\circ} 317$ Expediente $\mathrm{N}^{\circ} 706 / 13$ (28 de febrero de 2013).
Facultad de Ciencias Sociales de la UNICEN, dictada en la Unidad de Enseñanza Quequén. La misma estuvo desde su creación co-dirigida por la presidenta de la asociación civil Alfredo Marcenac (de la ciudad bonaerense de Necochea comprometida con la problemática de la tenencia y uso armas de fuego en contexto de violencia) y por Matta. De manera más puntual en el año 2014 se organizó una charla a mediadores del Ministerio Público y otros organismos, donde Godoy compartió por primera vez preguntas y líneas de interpretación antropológica sobre la práctica de la mediación y sobre la cuestión de la violencia interpersonal. En el año 2016, ofrecimos los tres autores de este artículo, con el aval de universidades y de organismos como el Ministerio de Justicia y Derechos Humanos de Salta un seminario de capacitación en la ciudad de Azul y en la ciudad de Salta dirigido a mediadores y al que llamamos "Antropología, derecho y mediación. Diálogos y perspectivas". En 2018 y 2019 Godoy y Matta dictaron el Seminario "Pedagogías de la Paz. Aportes de la Antropología" en la Especialidad en Mediación Educativa de la Universidad Nacional de Salta. En esa perspectiva de articular intereses y enfoques se incorporó hacia 2014 un mediador penal al proyecto de investigación que dirigía Godoy en la UNSa.

En Brasil, se organizó una actividad en el ámbito de la Escola de Serviço Social de la Universidad Federal de Rio de Janeiro, donde Mello es profesora. Se trata de la Oficina Antropologia e Políticas Públicas de administração de Conflitos: dilemas e perspectivas no Brasil e na Argentina. Nuestro objetivo fue un diálogo entre la Universidad e Instituciones y actores sociales involucrados con políticas públicas de administración de conflictos, en especial con la Mediación ${ }^{13}$.

En todas esas experiencias partimos de perspectivas teórico-metodológicas en la intersección entre las Ciencias Humanas y el Derecho, que indagan sobre las diferentes modalidades y sentidos que el instituto de la Mediación de Conflictos ha adquirido tanto desde el punto de vista de su dimensión práctica -judicial, comunitaria y penal- como desde el punto de vista de los campos empíricos en sociedades distintas como la brasilera y argentina. Así, pudimos debatir sobre las principales alternativas analíticas que la antropología jurídica viene desarrollando para el estudio de la mediación, focalizando en los conceptos, métodos y dimensiones que presentan desafíos y perspectivas sobre políticas públicas de administración de conflictos y seguridad pública en contextos de reformas estatales ${ }^{14}$. Algunas de estas

\footnotetext{
13 Tal iniciativa estaba inscripta en el Proyecto de investigación coordinado por Mello y en el cual Matta y Godoy son integrantes, titulado Análise comparada de categorias jurídicas em processos sociais de administração de conflitos: democracia, igualdade jurídica, cidadania e demanda por direitos - Edital Chamada INCT - MCTI/CNPq/CAPES/FAPs. Cadastrado en la Escola de Serviço SocialCFCH/UFRJ y en el INCT-InEAC-NEPEAC/UFF.

14 En dichos seminarios hemos desarrollado temas tales como la perspectiva antropológica y el concepto de cultura para pensar
} 
cuestiones serán retomadas en el apartado siguiente.

\subsection{Aproximaciones, superposiciones, ambivalencias y distancias entre la identidad del/la antropólogo/a y del/ la mediador/a}

Las articulaciones que describimos anteriormente fueron posibles por varias razones que hemos ido señalando respecto a las características institucionales de ese campo específico de la administración de conflictos. Ahora bien, también hay un conjunto amplio de saberes y prácticas propias de la antropología social que parecen resultar si no operativos, próximos cognitivamente con aquellos ámbitos favoreciendo esta aproximación. El dispositivo del "extrañamiento" y el relativismo cultural en la antropología como un modo de descentramiento del propio punto de vista y parámetro normativo para alcanzar mayores grados de comprensión de otredades parece asemejarse al principio de neutralidad, imparcialidad o multiparcialidad del mediador respecto a las partes en conflicto; la consideración por los aspectos contextuales y la historicidad de los conflictos; cierta atención en el conflicto en tanto proceso; la noción, sobre todo en la mediación comunitaria o bien con perspectiva territorial de la necesidad de aproximarse a los contextos reales en los que los conflictos se han desarrollado para realizar una intervención más adecuada; la preocupación metodológica por los modos de preguntar son algunos aspectos en que la antropología parece compartir cierta base conceptual y metodológica con la mediación.

En algunos casos el involucramiento con este campo de interés que comprendía tanto a la configuración del conflicto como sus alternativas de gestión fue tal que Matta reflexiona en otro trabajo (2020) que su objeto de estudio de alguna manera había absorbido parte de su identidad como antropólogo. Allí narra cómo lentamente comenzó a ser re-conocido en su ciudad y en otros contextos, sino como un mediador, como una especie de especialista local en resolución de conflicto. A la consulta referida de medios de comunicación, ONGs u organismos públicos se sumó la de compañeros de trabajo, familiares y amigos que transitaban alguna situación conflictiva.

También Godoy reconoce este proceso de cierto modo identitario y asociado a lo que en algún momento han llamado la creencia en la mediación (Godoy y Matta, 2018). Así, en la universidad y en espacios de relaciones personales se le consultó sobre esta alternativa ante conflictos anteriormente tratados de otro modo y ella misma consideró más de una vez adecuada esta

modelos de conflicto y de su administración; los aportes de la antropología jurídica al estudio de la mediación en cuanto a ubicarla en relación a diversos sistemas jurídicos y de control social; figuras de mediadores en sociedades no estatales; la noción de reconocimiento en la estructuración del conflicto y su resolución. Sobre las tensiones prácticas de la justicia restaurativa con el paradigma inquisitorial y la puja entre la promoción de la autonomía de los individuos y la reproducción de modelos normativos existentes. modalidad. Mello asimismo ha defendido en recientes publicaciones (Mello, 2018) a la mediación considerándola un método de administración de conflictos "no-violento" en un contexto de recrudecimiento del punitivismo estatal en Brasil y Latinoamérica.

Este proceso es particularmente alarmante en Brasil, con la instalación de un gobierno que ha reducido, e incluso impedido, iniciativas de esta naturaleza, diseminando discursos de intolerancia y de odio, que pueden, potencialmente, vaciar iniciativas de prevención de las diversas modalidades de violencia. El modo en que la población y los actores del campo de la mediación de conflictos vayan a lidiar con el contexto político actual, dependerá de factores que sólo el tiempo dirá cómo superar. Nuestra reflexión sobre el tema porta un esfuerzo en el sentido de insistir en las formas no violentas de gestionar el conflicto como principio eficaz de construcción de una sociedad que respete la diversidad social y cultural.

Podríamos preguntarnos cuánto este tipo de vínculo de proximidad con el campo exponía el trabajo etnográfico a ciertos riesgos en desmedro del extrañamiento. Pero de hecho hemos problematizado cómo la manifestación de cierto modo ritual del compromiso, apoyo o creencia en la mediación como método más eficaz y cercano al conflicto es un requerimiento implícito importante a la hora de formar parte o relacionarse con este universo con ciertos rasgos corporativos. Esta condición ha marcado también nuestras posibilidades de aproximación a ese campo desde la investigación antropológica y ha supuesto distintas tensiones. En distintas oportunidades hemos sentido cierta presión de acreditar una suerte de "conversión" a ciertos dogmas del sistema, que nos hemos encontrado defendiendo públicamente más de una vez; pero también ha generado una inercia para las tareas colaborativas de nuestro trabajo y el de Ixs mediadores. En cualquier circunstancia, estas tensiones en relación a nuestro rol en la aldea de la mediación pudieron ser reconvertidas mediante el ejercicio reflexivo en puntos de accesos a dichos universos sociales ampliando nuestras posibilidades de comprensión etnográfica de los mismos.

También estas relaciones estuvieron mediadas por ciertas afinidades ideológicas en cuanto a comprender la mediación en el marco de alternativas democratizadoras en el acceso a la justicia. Pero aquí pasamos a considerar las primeras distancias entre la perspectiva antropológica y la de la mediación. Nuestra insistente mirada sobre el propio dispositivo de mediación al interior de una economía política más amplia del control social y de administración de conflictos, lo que Laura Nader (1994) llama una "armonía coercitiva" puede contrastar hasta la incomodidad con una apreciación por parte algunos actores sociales del campo de la mediación, de la mediación como una práctica benevolente de por sí. En segundo lugar, un acentuado interés por el componente 
psíquico y conductual de los contendientes en algunas intervenciones en mediación y por el contrario, nuestro acento como antropólogos/as en la calidad de las relaciones sociales en el marco de vínculos de poder y de orden social más amplios para entender esos conflictos puede a simple vista parecer una perspectiva contrastante en ambos sistemas ${ }^{15}$. Cierto ímpetu civilizatorio en algunas intervenciones -el énfasis en el cambio de conducta para la pacificación del conflicto- puede resultar distante a la mirada analítica del conflicto. La advertencia del peligro de que como institución, la mediación se vuelva un marco cognitivo y normativo representado por los mediadores e impuesto a los mediados (De Leonardis y Pitch, 2010) también puede ser un punto incisivo.

Otras cuestiones como la observación de las continuidades entre el mundo de la mediación y el de los sistemas judiciales (Mello y Baptista, 2011); el señalamiento de la inconsistencia de algunas nociones como voluntariedad (Mello, 2016), autocomposición, neutralidad, escalada irracional en el contexto de la mediación; de los problemas de validación que acarrea la permeabilidad a saberes vinculados al new age (Carozzi, 1995), pusieron en tensión en reiteradas oportunidades nuestra mirada con la de los actores que adhieren a la mediación como a un movimiento. Cabe hacer notar que estas diferencias también a veces tensan el propio campo de la mediación y espacios de trabajo, generando mecanismos de separación y agrupación entre los mediadores.

Es interesante detenernos además en una reflexión sobre nuestra experiencia como docentes en el mundo de la formación y capacitación en la mediación. En éstas observamos cierto desequilibrio en las expectativas recíprocas asociadas a la fuerte demanda de estos universos de un conocimiento práctico sobre la gestión de los conflictos por sobre uno teórico sobre el tema. Los intereses teóricos en el conflicto y sus modos de administración que anima la empresa etnográfica no necesariamente coinciden con las preocupaciones prácticas que tienen los actores involucrados en su gestión, conllevando esto una persistente tensión entre interés analítico y practico de la gestión de conflictos.

Adicionalmente en distintas ocasiones los actores del campo de la mediación sostuvieron discursos antiacademicistas, críticos con cierto tipo de conocimiento abstracto y ciertas modalidades de enseñanza eruditas y expositivas. Y más aún, en algunos casos desconfiaban de enfoques demasiado recostados en una única disciplina. En este discurso la prevención de la violencia y la construcción de convivencia pacífica requerían de la interdisciplinariedad, dada la naturaleza sistémica, compleja y múltiple del conflicto para dicha concepción.

\footnotetext{
15 Si bien autores de la mediación como el noruego Johan Galtung, entiende que la violencia y el conflicto están asociados a componentes estructurales como la pobreza, el hambre, el analfabetismo y distintas carencias de carácter socio-económico.
}

Llama igualmente la atención la ambivalencia que implica este tono anti-académico y al mismo tiempo la penetración de la mediación en cursos de grado y posgrado en la universidad.

\section{Conclusiones}

En el presente artículo nos propusimos recuperar comparativamente algunos de nuestros avances de investigación respecto al desarrollo del campo de la Mediación de conflictos en Argentina y Brasil, vinculando su emergencia y desarrollo a las características específicas de la estatalidad que lo comprende, es decir, analizando el movimiento de mediación en las articulaciones, discontinuidades y esfuerzos de autonomización de sus actores respecto a distintos niveles gubernamentales. El campo de la mediación se revela así como una malla institucional que pretende cada vez más extensión, que une además el campo jurídico con otros campos de la administración pública, gobiernos nacionales, provinciales -o estaduales- y municiapales, ONGs y universidades y que se encuentra marcado por el dinamismo y la disputa. Allí nuestro lugar como antropólogxs resultó al mismo tiempo consonante como perturbador.

El hecho de que la mediación sea un movimiento que no se reduce a su forma estatal moldea tanto las formas estatales de la mediación como las posibilidades y dinámicas de la investigación etnográfica sobre dicho universo. Así, por ejemplo, la existencia de redes por fuera del Estado que articulan múltiples actores vinculados a la mediación ofrece una malla capaz de contener los vaivenes del trabajo de los mediadores en el Estado. Tomando como caso, cuando los cambios en las administraciones remueven a personal involucrado en programas de mediación estos pueden encontrar/ construir alternativas laborales en el marco de esta red no estatal hasta que nuevos cambios posibiliten el reingreso al mismo. En nuestras experiencias de investigación tejimos alianzas con actores que resultaron ocasionalmente estatales (revelando esto un aspecto de la estatalidad de la mediación) pero que una vez fuera de estas órbitas seguían siendo actores de un movimiento con capacidad de disputar, reclamar y participar en otros ámbitos estatales.

A lo largo de este trabajo quisimos mostrar cómo el trabajo de campo antropológico se asienta en alianzas con actores del campo por lo que la suerte del propio trabajo de investigación es reveladora de la dinámica y naturaleza del propio campo, en este caso, el de la mediación dentro del más amplio campo estatal de la administración de conflictos. Así las tensiones y compromisos entre nuestro trabajo como antropólogos y el mundo de la mediación nos permitieron reflexionar sobre dicho universo reconociendo aspectos que incluso no formaron parte de nuestros intereses iniciales de indagación. El lugar del Estado en la mediación es una 
de estas cuestiones emergentes y pude ser pensado a partir de un ejercicio de reflexividad (Guber 2001) sobre las propias experiencias etnográficas.

Salta, Olavarría, Rio de Janeiro, 27 de diciembre de 2019

\section{Bibliografía}

Amorim, M. S.; Kant de Lima, R.; Burgos, M. B. (Orgs.) (2003). Juizados Especiais Criminais, sistema judicial e sociedade no Brasil: ensaios interdisciplinares. Niterói: Intertexto.

Azevedo, Rodrigo Guiringhelli de. (2000). Informalização da justiça e controle social. Estudo sobre a implantação dos Juizados Especiais Criminais em Porto Alegre. São Paulo: IBCCRIM.

Bourdieu, P. (1990). Algunas propiedades de los campos. Sociología y cultura. México: Conaculta. pp. 135-141.

Bourdieu, P. (1997). Espíritus de Estado. Génesis y estructura del campo burocrático. Razones prácticas. Sobre la teoría de la acción. Barcelona: Anagrama.

Bourdieu, P. (2014). Sobre el Estado. Cursos en el Collége de France (1989-1992). España: Anagrama.

Bourdieu, P. y Wacquant L., (1995). La lógica de los campos. Respuestas. Por una sociología reflexiva. México: Grijalbo. Pp. 63-68.

Bush, R. A. B., y Folger, J. P. (1996). La promesa de la mediación. Cómo afrontar el conflicto a través del fortalecimiento y el reconocimiento de los otros. Barcelona: Granica.

Cardoso de Oliveira, L. R. (2004). Honor, dignidad y reciprocidad. Cuadernos de Antropología Social. Buenos Aires. Universidad de Buenos Aires, 2004.

Carozzi, M. J. (1995). Definiciones de la New Age desde las ciencias sociales. Boletín de lecturas sociales $y$ económicas, 2(5), 19-24.

De Leonardis, O.; Pitch, T. (2010). Un mundo horizontal. Las retóricas y las prácticas contemporáneas de la mediación. En Sozzo, M. (Comp.). Por una sociología crítica del control social: ensayos en honor a Juan $S$. Pegorano. (pp. 79-96). Buenos Aires: Del Puerto.

Fasano, P. (2014). Enredada. Dilemas sobre el proceso etnográfico de investigación de un chisme y su publicación. Prácticas etnográficas. Ejercicios de reflexividad de antropólogas de campo. Guber, R.(comp.) IDES: Miño y Dávila, 156-182.

Fassin, D. (2003). Gobernar por los cuerpos, políticas de reconocimiento hacia los pobres y los inmigrantes en Francia. Cuadernos de antropología social, (17), 49-78.

Fisher, R., y Ury, W. (1991). Getting to Yes: Negotiating an agreement without giving in. New York: A Perigee Book/Penguin Group.

Fleischer, S. (2007). Antropólogos anfíbios? Alguns comentários sobre a relação entre Antropología e intervenção no Brasill. Revista Anthropológicas, Ano 11, Vol. 18(1):37-70.

Fleming, R. W. (1985). Reflections on the ADR Movement. Clev. St. L. Rev., 34, 519.

Foucault, Michel (2006). Clase del 1 ro de febrero de 1978. Seguridad, territorio, población (pp. 109-138). Buenos Aires: Fondo de Cultura Económica.

Godoy, M. I. (2016a). Delito, conflicto: sensibilidades legales y trama institucional en el campo de la mediación penal en Salta, Argentina. Revista del Museo de Antropología, 9(1), 57-68.

Godoy, M. I. (2016b). De la denuncia a la mediación: sensibilidades legales y usos del derecho en conflictos de proximidad. Corpus. Archivos virtuales de la alteridad americana, 6(2).

Godoy, M. I., \& Matta, J. P. (2018). Creencia y eficacia en la profesión del Mediador de conflictos. Una aproximación desde la teoría antropológica de la magia. Potencialidades e incertezas de formas não violentas de administração de conflitos no Brasil e na Argentina. Porto Alegre: Evangraf/Palmarinca.

Guber, R. (2001). La etnografía. Método, campo y reflexividad. Buenos Aires: Grupo Editorial Norma.

Manzano, V., Ramos, A. (2015). Procesos de movilización y de demandas colectivas: estudios y modos de abordar lo político en la vida social. Identidades; Comodoro Rivadavia; p. $1-25$

Matta, J. P. (2016). Entre vecinos eso no se hace. Sentidos de justicia y de vecindad en el marco de un dispositivo institucional de administración de conflictos. Antípoda. Revista de Antropología y Arqueología, (24), 55-71.

Matta, J. P., y Godoy, M. I. (2016). El movimiento de Mediación en Argentina: procesos, tensiones y afirmaciones. Revista de Estudos e Pesquisas sobre as Américas, 57.

Matta, J. P. (2020). El trabajo antropológico en el mundo de la mediación de conflictos. Consideraciones para un diálogo posible. Administração de Conflitos, Espaço Público e Sociabilidades Urbanas em perspectiva comparada, Mello, K.S.S e Farias, P.S. En prensa. 
McEwen, C. A. (1988). The ADR Movement: Theoretical Aspects and Practical Protential. Me. L. Rev., 40, 367.

Mello, K. S. S. (2020 prensa). Mediação Judicial de Conflitos: balanço e potencialidade. Administração de Conflitos, Espaço Público e Sociabilidades Urbanas em perspectiva comparada, Mello, K.S.S e Farias, P.S.

Mello, K. S. S., Lupetti Baptista, B. y Filpo, K.P.L. (org.) (2018). Potencialidades e incertezas de formas não violentas de administração de conflitos no Brasil e na Argentina. Porto Alegre: Evangraf/Palmarinca.

Mello, K. S. S. (2016). Mediação de conflitos e voluntariedade: olhares cruzados entre Rio de Janeiro e Buenos Aires. Antropolitica Revista Contemporanea de Antropologia, 1(40).

Mello, K. S. S., \& Baptista, B. G. L. (2011). Mediação e conciliação no judiciário: dilemas e significados. DilemasRevista de Estudos de Conflito e Controle Social, 4(1), 97-122.

Merry, S. E. (1995). "Sorting Out Popular Justice". En: Merry, S. E \& Milmer, N. (eds.). The Possibility of Popular Justice. A Case Study of Community Mediation in the United States. The University of Michigan Press, Michigan, USA

Muzzopappa, E. \& Villalta, C. (2011). Los documentos como campo. Reflexiones teórico-metodológicas sobre un enfoque etnográfico de archivos y documentos estatales. Revista Colombiana de Antropología, 47(1), 13-42.

Nader, L. (1994). Ideología armónica. Justicia y control en un pueblo de la montaña zapoteca, IOC-CIESAS, México.

Rosenberg M. (2006). Comunicación no violenta. Un lenguaje de vida. Buenos Aires. Gran Aldea Editores.

Simião, D. S., Duarte, V. B., Carvalho, N. D., \& Davis, P. G. (2010). Sentidos de justiça e reconhecimento em formas extrajudiciais de resolução de conflitos em Belo Horizonte. En Roberto Kant de Lima, R. Eilbaum, L. y Pires L. (orgs.) Conflitos, direitos e moralidades em perspectiva comparada. Rio de Janeiro: Garamond, 1.

Simião, D. S. (2015). As donas da palavra: gênero, justiça e a invenção da violência doméstica em Timor-Leste. Brasília: Editora UnB.

Sinhoretto, J. (2010). Campo estatal de administração de conflitos: múltiplas intensidades da justiça. Anuário Antropológico, (II), 109-123.

Sinhoretto, J. (2011). Justiça perto do povo: reforma e gestão de conflitos, São Paulo: Alameda.

Thomas, M. S. (2016). Theorizing Meditation: Lessons Learned from Legal Anthropology. Utrecht L. Rev., 12, 50. 